\title{
The Role of Dihydrogen Oxide in Dental Practice
}

\author{
Crasspy McTabernacle*, MSc, DDS, PhD, Attila T.H.E. Hun, DDS, PhD, MSc, Beau Peep, PhD, \\ DDS, M. Arse, Cupid Stunt, PhD, DDS
}

Electric Jockstrap Research Institute, University of Neasden, London, United Kingdom

*Corresponding Author: Crasspy McTabernacle, MSc, DDS, PhD, Electric Jockstrap Research Institute, University of Neasden, London, United Kingdom, Email: baldgit58@hotmail.com

\begin{abstract}
The role of dihydrogen oxidehas recently been brought to the attention of the dental profession. This short article examines the origin, history, recent uses and future possibilities for this compound.
\end{abstract}

\section{INTRODUCTION AND LITERATURE REVIEW}

Dihydrogen oxide is a common compound in the earth's atmosphere and in soils, rocks and other sedimentary deposits. It is formed as the reaction product of two common elements, hydrogen and oxygen and in nature it may be found with varying levels of trace elements such as cadmium, fluorine, molybdenum, manganese, chlorine and others. Its use in industrial processes is widespread, such as in the cloth manufacturing industry and in food processing. It is extensively used in the production of electricity and in nuclear power plants. It can exist in three forms - gaseous, liquid and solid, depending on the temperature.

In the domestic situation, it is used as a cleaning agent, and also in the preparation of foodstuffs. A regular supply is a mark of how developed a country is on the UN international index of development.

More recently its role in medicine and dentistry has highlighted many of its useful properties. It can be used as a solvent, and as a cleaning agent in many clinical processes. Purified forms of this compound can be used in clinical medicine and various elements can be added to it such as sodium chloride to ensure isomolarity. In the administration of drugs it can be used to dissolve various compounds and ingested, it can be injected using a syringe and needle, or it can be used in a vapourised form such as in aerosols. In dental practice, its use in cleansing the oral cavity is most prominent, while the introduction of various dental cements relies on its addition to a powder to transform the components into a flowable material.

Antibacterial properties of dihydrogen oxide remain equivocal. In certain cases it can be useful in gross cleaning of the infected area to remove large visible amounts of contaminatns, but as an antibiotic it is not particularly effective.

Dihydrogen oxide does have a number of side effects. If ingested in too large quantities it can result in excessive micturition and an increase in blood pressure. It forms a large proportion of alcoholic drinks and alcohol addiction is a problem in many countries. It has been found that alcoholics have a severe negative reaction to the prospect of ingesting significant quantities of this compound and rehabilitation is often necessary in these cases. Its presence in seawater is very high and shipwrecked sailors have died from drinking too much seawater.

All dentists should make use of this common material and ensure that their daily practice is geared towards the efficient use and disposal in the dental surgery.

\section{REFERENCES}

[1] Walker, J. (1999) The use of hydrogen dihydroxide in the production of ethanol derivatives. The Journal of Distillation Research, 47, 19-23 
[2] Grant, W. (2017) Awa' ye Sassenach bastards. Can't ye see I'm pished? Research in Addiction, 93, 12-1042
[3] Glenfiddich, G. (1997). On the proportions of hydrogen dihydroxide in ethanol derivatives at low temperatures. Archives of Fluid Dynamics, 17, 4-9

Citation: Crasspy McTabernacle, Attila T.H.E. Hun, Beau Peep, Cupid Stunt. "The Role of Dihydrogen Oxide in Dental Practice". ARC Journal of Dental Science. 2021; 6(1):1-2. DOI: https://doi.org/10.20431/24560030.0601001.

Copyright: (C) 2021 Authors. This is an open-access article distributed under the terms of the Creative Commons Attribution License, which permits unrestricted use, distribution, and reproduction in any medium, provided the original author and source are credited. 